Louisiana State University

LSU Digital Commons

8-20-2018

\title{
Search for GeV Gamma-Ray Counterparts of Gravitational Wave Events by CALET
}

\author{
O. Adriani \\ Università degli Studi di Firenze \\ Y. Akaike \\ University of Maryland, Baltimore County (UMBC) \\ K. Asano \\ The University of Tokyo \\ Y. Asaoka \\ Waseda University \\ M. G. Bagliesi \\ Università degli Studi di Siena
}

See next page for additional authors

Follow this and additional works at: https://digitalcommons.Isu.edu/physics_astronomy_pubs

\section{Recommended Citation}

Adriani, O., Akaike, Y., Asano, K., Asaoka, Y., Bagliesi, M., Berti, E., Bigongiari, G., Binns, W., Bonechi, S., Bongi, M., Brogi, P., Buckley, J., Cannady, N., Castellini, G., Checchia, C., Cherry, M., Collazuol, G., Felice, V., Ebisawa, K., Fuke, H., Guzik, T., Hams, T., Hareyama, M., Hasebe, N., Hibino, K., Ichimura, M., loka, K., Ishizaki, W., Israel, M., Kasahara, K., Kataoka, J., Kataoka, R., \& Katayose, Y. (2018). Search for GeV Gamma-Ray Counterparts of Gravitational Wave Events by CALET. Astrophysical Journal, 863 (2) https://doi.org/10.3847/1538-4357/aad18f

This Article is brought to you for free and open access by the Department of Physics \& Astronomy at LSU Digital Commons. It has been accepted for inclusion in Faculty Publications by an authorized administrator of LSU Digital Commons. For more information, please contact ir@lsu.edu. 


\section{Authors}

O. Adriani, Y. Akaike, K. Asano, Y. Asaoka, M. G. Bagliesi, E. Berti, G. Bigongiari, W. R. Binns, S. Bonechi, M. Bongi, P. Brogi, J. H. Buckley, N. Cannady, G. Castellini, C. Checchia, M. L. Cherry, G. Collazuol, V. Di Felice, K. Ebisawa, H. Fuke, T. G. Guzik, T. Hams, M. Hareyama, N. Hasebe, K. Hibino, M. Ichimura, K. Ioka, W. Ishizaki, M. H. Israel, K. Kasahara, J. Kataoka, R. Kataoka, and Y. Katayose 


\section{Search for GeV Gamma-Ray Counterparts of Gravitational Wave Events by CALET}

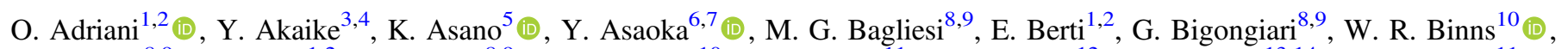
S. Bonechi ${ }^{8,9}$, M. Bongi ${ }^{1,2}$ (1) , P. Brogi ${ }^{8,9}$, J. H. Buckley ${ }^{10}$, N. Cannady $^{11}$, G. Castellini ${ }^{12}$, C. Checchia ${ }^{13,14}$, M. L. Cherry ${ }^{11}$ (1), G. Collazuol ${ }^{13,14}$, V. Di Felice ${ }^{15,16,17}$ (1), K. Ebisawa ${ }^{18}$ (D) H. Fuke ${ }^{18}$, T. G. Guzik ${ }^{11}$, T. Hams ${ }^{3,19}$, M. Hareyama ${ }^{20}$, N. Hasebe ${ }^{6}$,

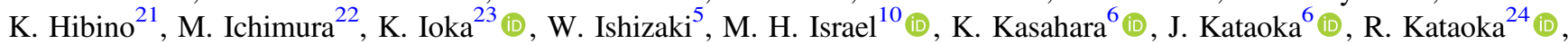
Y. Katayose $^{25}$, C. Kato ${ }^{26}$, N. Kawanaka ${ }^{27,28}$ (1) , Y. Kawakubo ${ }^{29}$, H. S. Krawczynski ${ }^{10}$ (1), J. F. Krizmanic ${ }^{3,19}$, K. Kohri ${ }^{30}$, T. Lomtadze 9 , P. Maestro ${ }^{8,9}$, P. S. Marrocchesi ${ }^{8,9}$, A. M. Messineo ${ }^{9,31}$, J. W. Mitchell ${ }^{4}$, S. Miyake ${ }^{32}$, A. A. Moiseev ${ }^{19,33}$, K. Mori ${ }^{6,18}$, M. Mori ${ }^{34}$ (1) , N. Mori ${ }^{2}$ (1) H. M. Motz ${ }^{35}$, K. Munakata ${ }^{26}$ (1) H. Murakami ${ }^{6}$, S. Nakahira ${ }^{36}$, J. Nishimura ${ }^{18}$, G. A. de $\mathrm{Nolfo}^{37}$, S. Okuno ${ }^{21}$, J. F. Ormes ${ }^{38}$, S. Ozawa ${ }^{6}$, L. Pacini ${ }^{1,2,12}$, F. Palma ${ }^{15,16}$, P. Papini ${ }^{2}$, A. V. Penacchioni ${ }^{8,39}$ B. F. Rauch $^{10}$, S. B. Ricciarini ${ }^{2,12}$ (1) , K. Sakai ${ }^{3,19}$, T. Sakamoto ${ }^{29}$, M. Sasaki ${ }^{19,33}$, Y. Shimizu ${ }^{21}$, A. Shiomi ${ }^{40}$, R. Sparvoli ${ }^{15,16}$ (1), P. Spillantini ${ }^{1}$, F. Stolzi ${ }^{8,9}$, J. E. Suh ${ }^{8,9}$ (1), A. Sulaj ${ }^{8,9}$, I. Takahashi ${ }^{41}$, M. Takayanagi ${ }^{18}$, M. Takita ${ }^{5}$, T. Tamura ${ }^{21}$, N. Tateyama ${ }^{21}$, T. Terasawa ${ }^{36}$, H. Tomida ${ }^{18}$, S. Torii ${ }^{6,7,42}$, Y. Tsunesada ${ }^{43}$ (D), Y. Uchihori ${ }^{44}$, S. Ueno ${ }^{18}$, E. Vannuccini ${ }^{2}$, J. P. Wefel ${ }^{11}$, K. Yamaoka ${ }^{45}$, S. Yanagita ${ }^{46}$, A. Yoshida ${ }^{29}$, and K. Yoshida ${ }^{47}$

(CALET Collaboration)

${ }^{1}$ Department of Physics, University of Florence, Via Sansone, 1 I-50019 Sesto, Fiorentino, Italy

${ }^{2}$ INFN Sezione di Florence, Via Sansone, 1 I-50019 Sesto, Fiorentino, Italy

${ }^{3}$ Department of Physics, University of Maryland, Baltimore County, 1000 Hilltop Circle, Baltimore, MD 21250, USA

${ }_{4}^{4}$ Astroparticle Physics Laboratory, NASA/GSFC, Greenbelt, MD 20771, USA

${ }^{5}$ Institute for Cosmic Ray Research, The University of Tokyo, 5-1-5 Kashiwa-no-Ha, Kashiwa, Chiba 277-8582, Japan

${ }^{6}$ Waseda Research Institute for Science and Engineering, Waseda University, 3-4-1 Okubo, Shinjuku, Tokyo 169-8555, Japan; yoichi.asaoka@ aoni.waseda.jp

${ }^{7}$ JEM Utilization Center, Human Spaceflight Technology Directorate, Japan Aerospace Exploration Agency, 2-1-1 Sengen, Tsukuba, Ibaraki 305-8505, Japan

${ }^{8}$ Department of Physical Sciences, Earth and Environment, University of Siena, via Roma 56, I-53100 Siena, Italy ${ }^{9}$ INFN Sezione di Pisa, Polo Fibonacci, Largo B. Pontecorvo, 3 I-56127 Pisa, Italy

${ }^{10}$ Department of Physics, Washington University, One Brookings Drive, St. Louis, MO 63130-4899, USA

${ }^{11}$ Department of Physics and Astronomy, Louisiana State University, 202 Nicholson Hall, Baton Rouge, LA 70803, USA

${ }^{12}$ Institute of Applied Physics (IFAC), National Research Council (CNR), Via Madonna del Piano, 10, I-50019 Sesto, Fiorentino, Italy

${ }^{13}$ Department of Physics and Astronomy, University of Padova, Via Marzolo, 8, I-35131 Padova, Italy ${ }^{14}$ INFN Sezione di Padova, Via Marzolo, 8, I-35131 Padova, Italy

${ }^{15}$ University of Rome "Tor Vergata," Via della Ricerca Scientifica 1, I-00133 Rome, Italy

${ }^{16}$ INFN Sezione di Rome "Tor Vergata," Via della Ricerca Scientifica 1, I-00133 Rome, Italy

${ }^{17}$ Space Science Data Center-Agenzia Spaziale Italiana, via del Politecnico, s.n.c., I-00133, Roma, Italy

${ }^{18}$ Institute of Space and Astronautical Science, Japan Aerospace Exploration Agency, 3-1-1 Yoshinodai, Chuo, Sagamihara, Kanagawa 252-5210, Japan

${ }^{19}$ CRESST and Astroparticle Physics Laboratory NASA/GSFC, Greenbelt, MD 20771, USA

${ }^{20}$ St. Marianna University School of Medicine, 2-16-1, Sugao, Miyamae-ku, Kawasaki, Kanagawa 216-8511, Japan

${ }^{21}$ Kanagawa University, 3-27-1 Rokkakubashi, Kanagawa, Yokohama, Kanagawa 221-8686, Japan

${ }^{22}$ Faculty of Science and Technology, Graduate School of Science and Technology, Hirosaki University, 3, Bunkyo, Hirosaki, Aomori 036-8561, Japan

${ }^{23}$ Yukawa Institute for Theoretical Physics, Kyoto University, Kitashirakawa Oiwakecho, Sakyo, Kyoto 606-8502, Japan

${ }^{24}$ National Institute of Polar Research, 10-3, Midori-cho, Tachikawa, Tokyo 190-8518, Japan

${ }^{25}$ Faculty of Engineering, Division of Intelligent Systems Engineering, Yokohama National University, 79-5 Tokiwadai, Hodogaya, Yokohama 240-8501, Japan

${ }^{26}$ Faculty of Science, Shinshu University, 3-1-1 Asahi, Matsumoto, Nagano 390-8621, Japan

${ }^{27}$ Hakubi Center, Kyoto University, Yoshida Honmachi, Sakyo-ku, Kyoto, 606-8501, Japan

${ }^{28}$ Department of Astronomy, Graduate School of Science, Kyoto University, Kitashirakawa Oiwake-cho, Sakyo-ku, Kyoto, 606-8502, Japan

29 College of Science and Engineering, Department of Physics and Mathematics, Aoyama Gakuin University, 5-10-1 Fuchinobe, Chuo, Sagamihara, Kanagawa 252-5258, Japan

${ }^{30}$ Institute of Particle and Nuclear Studies, High Energy Accelerator Research Organization, 1-1 Oho, Tsukuba, Ibaraki, 305-0801, Japan

${ }^{31}$ University of Pisa, Polo Fibonacci, Largo B. Pontecorvo, 3 I-56127 Pisa, Italy

${ }^{32}$ Department of Electrical and Electronic Systems Engineering, National Institute of Technology, Ibaraki College, 866 Nakane, Hitachinaka, Ibaraki $312-8508$ Japan

${ }^{33}$ Department of Astronomy, University of Maryland, College Park, MD 20742, USA

${ }^{34}$ Department of Physical Sciences, College of Science and Engineering, Ritsumeikan University, Shiga 525-8577, Japan; morim @ fc.ritsumei.ac.jp

${ }^{35}$ Global Center for Science and Engineering Programs, Waseda University, 3-4-1 Okubo, Shinjuku, Tokyo 169-8555, Japan

${ }^{36}$ RIKEN, 2-1 Hirosawa, Wako, Saitama 351-0198, Japan

${ }^{37}$ Heliospheric Physics Laboratory, NASA/GSFC, Greenbelt, MD 20771, USA

${ }^{38}$ Department of Physics and Astronomy, University of Denver, Physics Building, Room 211, 2112 East Wesley Avenue, Denver, CO 80208-6900, USA

${ }^{39}$ ASI Science Data Center (ASDC), Via del Politecnico snc, I-00133 Rome, Italy

${ }^{40}$ College of Industrial Technology, Nihon University, 1-2-1 Izumi, Narashino, Chiba 275-8575, Japan

${ }^{41}$ Kavli Institute for the Physics and Mathematics of the Universe, The University of Tokyo, 5-1-5 Kashiwanoha, Kashiwa, 277-8583, Japan

${ }^{42}$ School of Advanced Science and Engineering, Waseda University, 3-4-1 Okubo, Shinjuku, Tokyo 169-8555, Japan

${ }^{43}$ Division of Mathematics and Physics, Graduate School of Science, Osaka City University, 3-3-138 Sugimoto, Sumiyoshi, Osaka 558-8585, Japan

${ }^{44}$ National Institutes for Quantum and Radiation Science and Technology, 4-9-1 Anagawa, Inage, Chiba 263-8555, Japan ${ }^{45}$ Nagoya University, Furo, Chikusa, Nagoya 464-8601, Japan

${ }^{46}$ College of Science, Ibaraki University, 2-1-1 Bunkyo, Mito, Ibaraki 310-8512, Japan

${ }^{47}$ Department of Electronic Information Systems, Shibaura Institute of Technology, 307 Fukasaku, Minuma, Saitama 337-8570, Japan Received 2018 March 29; revised 2018 July 2; accepted 2018 July 3; published 2018 August 20 


\begin{abstract}
We present the results of searches for gamma-ray counterparts of the LIGO/Virgo gravitational wave events using CALorimetric Electron Telescope (CALET) observations. The main instrument of CALET, CALorimeter (CAL), observes gamma-rays from $\sim 1 \mathrm{GeV}$ up to $10 \mathrm{TeV}$ with a field of view (FOV) of nearly $2 \mathrm{sr}$. In addition, the CALET gamma-ray burst monitor views $\sim 3 \mathrm{sr}$ and $\sim 2 \pi$ sr of the sky in the $7 \mathrm{keV}-1 \mathrm{MeV}$ and the $40 \mathrm{keV}-20 \mathrm{MeV}$ bands, respectively, by using two different crystal scintillators. The CALET observations on the International Space Station started in 2015 October, and here we report analyses of events associated with the following gravitational wave events: GW151226, GW170104, GW170608, GW170814, and GW170817. Although only upper limits on gamma-ray emission are obtained, they correspond to a luminosity of $10^{49} \sim 10^{53} \mathrm{erg} \mathrm{s}^{-1}$ in the GeV energy band depending on the distance and the assumed time duration of each event, which is approximately on the order of luminosity of typical short gamma-ray bursts. This implies that there will be a favorable opportunity to detect highenergy gamma-ray emission in further observations if additional gravitational wave events with favorable geometry will occur within our FOV. We also show the sensitivity of CALET for gamma-ray transient events, which is on the order of $10^{-7} \mathrm{erg} \mathrm{cm}^{-2} \mathrm{~s}^{-1}$ for an observation of $100 \mathrm{~s}$ in duration.
\end{abstract}

Key words: gamma rays: general - gravitational waves - methods: observational

\section{Introduction}

The discovery of gravitational wave $(\mathrm{GW})$ events using laser interferometers by the LIGO and Virgo Scientific Collaborations (Abbott et al. 2016a) was an epoch-making development following the prediction of the existence of gravitational waves by Einstein $(1916,1918) 100$ years earlier. GW events are thought to be produced in the last stage of merging compact binaries, and electromagnetic counterparts of these events have been extensively discussed by many authors. Merging neutron star-neutron star (NS-NS) binaries and neutron star-black hole (NS-BH) binaries are thought to emit a significant amount of electromagnetic radiation (e.g., Phinney 2009; Rosswog 2015; Fernández \& Metzger 2016), while it is often assumed that gravitational wave events resulting from the merger of stellar-mass black holes are unlikely to produce electromagnetic counterparts (e.g., de Mink \& King 2017).

Since the study of GW events is in the early stages, it is needless to say that the multimessenger approach is exceedingly important in order to understand the nature of the production mechanisms. In particular, mergers of NS-NS binaries are hypothesized to be a possible origin of short gamma-ray bursts (sGRBs; e.g., Goodman 1986; Pacyński 1986; Eichler et al. 1989; Narayan et al. 1992; Mochkovitch et al. 1993) and thus the observation in the gamma-ray energy region is essential to understanding the connection between sGRBs to GW events.

We summarize the characteristics of six GW events during the first and second advanced LIGO-Virgo observing runs in Table 1 with inferred parameters. We then report the analysis of CALorimetric Electron Telescope (CALET)/CAL observations corresponding to these gravitational events (except GW150914, which occurred before the start of CALET operations) in the gamma-ray energy region as briefly summarized in Table 1.

\section{Observation and Analysis}

\subsection{CALET Observation}

The CALET mission (Torii \& for the CALET Collaboration 2015) was launched and placed on the Japanese Experiment Module-Exposed Facility of the International Space Station (ISS) in 2015 August. At the LIGO trigger time of GW150914, CALET was in its commissioning phase and no observational data were available. It was fully functional at the trigger times of GW151226, GW170104, GW170608, GW170814, and GW170817.

There are two scientific instruments on board CALET: (1) the Calorimeter $(\mathrm{CAL})$, the main instrument, is a 30 radiation length deep calorimeter that can observe high-energy electrons in the energy range of $\sim 1 \mathrm{GeV}-\sim 20 \mathrm{TeV}$, protons, helium, and heavy nuclei in the energy range of $\sim 10 \mathrm{GeV} \sim 1000 \mathrm{TeV}$ and gamma-rays in the energy range of $\sim 1 \mathrm{GeV}-\sim 10 \mathrm{TeV}$. The field of view (FOV) of CAL extends to $\sim 45^{\circ}$ from the zenith direction. For gamma-rays, the energy resolution and the angular resolution are estimated to be $3 \%$ and 0.4 , respectively, at $10 \mathrm{GeV}$ (Mori \& for the CALET Collaboration 2013; Cannady \& for the CALET Collaboration 2017, 2018). CAL consists of three main components: the CHarge Detector (CHD), the IMaging Calorimeter (IMC), and the Total AbSorption Calorimeter (TASC) (Figure 1). CHD is made up of a set of $X$ - and $Y$-direction arrays of 14 plastic scintillator paddles $(32 \mathrm{~mm} \times 10 \mathrm{~mm} \times 450 \mathrm{~mm})$; IMC is composed of eight layers of $X$ - and $Y$-direction arrays of 448 scintillation fibers (SciFi, $1 \mathrm{~mm} \times 1 \mathrm{~mm} \times 448 \mathrm{~mm}$ ) separated by tungsten plates with a total thickness of 3 radiation lengths $\left(X_{0}\right)$; and TASC is made of 6 layers of $X$ - and $Y$-arrays of 16 lead tungstate $\left(\mathrm{PbWO}_{4}\right.$ or $\left.\mathrm{PWO}\right)$ scintillation crystals $(19 \mathrm{~mm} \times 20 \mathrm{~mm} \times$ $326 \mathrm{~mm}$ ) with a total thickness of $27 X_{0}$. (See Asaoka et al. 2017 for details.) The performance of CAL for gamma-rays and initial results for steady gamma-ray sources are described in Cannady $\&$ for the CALET Collaboration (2017, 2018). (2) A companion instrument, the CALET Gamma-ray Burst Monitor (CGBM), monitors gamma-ray bursts (GRBs) using two different kinds of crystal scintillators $\left(\mathrm{LaBr}_{3}(\mathrm{Ce})\right.$ and $\left.\mathrm{Bi}_{4} \mathrm{Ge}_{3} \mathrm{O}_{12}(\mathrm{BGO})\right)$ to cover a wide energy range $(7 \mathrm{keV}-20 \mathrm{MeV}$; Yamaoka \& for the CALET Collaboration 2013). Results from CGBM are presented separately (Yamaoka \& for the CALET Collaboration 2017).

We use two trigger modes of CAL for gamma-ray analysis: a low-energy gamma-ray (LE- $\gamma$ ) mode with an energy threshold $\sim 1 \mathrm{GeV}$ used at low geomagnetic latitudes and following a CGBM burst trigger, and a high-energy (HE) mode with a threshold $\sim 10 \mathrm{GeV}$ used in normal operation for all particles irrespective of geomagnetic latitude (Asaoka et al. 2018). Around the trigger time of GW151226, between 03:30 and 03:43 UT, CAL was collecting regular scientific data under the LE- $\gamma$ mode. The high voltages supplied to photomultipliers of CGBM detectors were set at the nominal values around 03:22 
Table 1

Summary of CALET Observations of Gravitational Events Reported by the Virgo and LIGO Scientific Collaborations (BH: black hole, NS: Neutron Star) and Representative Results from CALET Observation (See the Text for Other Time Windows)

\begin{tabular}{|c|c|c|c|c|c|c|c|c|c|}
\hline \multirow{3}{*}{$\begin{array}{l}\text { GW } \\
\text { event }\end{array}$} & \multirow{3}{*}{$\begin{array}{c}\text { Time } \\
T_{0} \\
\text { (UTC) }\end{array}$} & \multirow{3}{*}{$\begin{array}{c}\text { Location } \\
\text { area } \\
\left(\operatorname{deg}^{2}\right)\end{array}$} & \multirow{3}{*}{$\begin{array}{r}\text { Luminosity } \\
\text { distance } \\
(\mathrm{Mpc})\end{array}$} & \multirow{3}{*}{$\begin{array}{l}\text { Event } \\
\text { Type }\end{array}$} & \multirow{3}{*}{ References } & \multicolumn{4}{|c|}{ CALET Results [Time Window] } \\
\hline & & & & & & \multirow{2}{*}{ Mode } & \multirow{2}{*}{$\begin{array}{l}\text { Summed } \\
\text { LIGO } \\
\text { probability }\end{array}$} & \multicolumn{2}{|c|}{ Upper Limits (90\% C.L.) } \\
\hline & & & & & & & & $\begin{array}{l}\text { Energy Flux } \\
\left(\mathrm{erg} \mathrm{cm}^{-2} \mathrm{~s}^{-1}\right)\end{array}$ & $\begin{array}{l}\text { Luminosity } \\
\left(\mathrm{erg} \mathrm{s}^{-1}\right)\end{array}$ \\
\hline GW150914 & $\begin{array}{r}2015 \text { Sep } 14 \\
09: 50: 45\end{array}$ & 600 & $440_{-180}^{+160}$ & $\mathrm{BH}-\mathrm{BH}$ & (a) & & \multicolumn{3}{|c|}{ Before operation } \\
\hline GW151226 & 2015 Dec 26 & 850 & $440_{-190}^{+180}$ & $\mathrm{BH}-\mathrm{BH}$ & (b) & LE & $15 \%$ & \multicolumn{2}{|c|}{$\left[T_{0}-525 \mathrm{~s}, T_{0}+211 \mathrm{~s}\right]$} \\
\hline & 03:38:53 & & & & & & & $\begin{array}{c}9.3 \times 10^{-8} \\
\end{array}$ & {$\left[T_{0}-60 \mathrm{~s}, T_{0}+60 \mathrm{~s}\right]$} \\
\hline GW170104 & $\begin{array}{r}2017 \text { Jan } 04 \\
10: 11: 58\end{array}$ & 1200 & $880_{-390}^{+430}$ & BH-BH & (c) & $\mathrm{HE}$ & $30 \%$ & $\begin{array}{l}{\left[T_{0}-60 \mathrm{~s}, T_{0}+\right.} \\
6.4 \times 10^{-6}\end{array}$ & $\begin{array}{l}\text { s] } \\
6.2 \times 10^{50}\end{array}$ \\
\hline GW170608 & $\begin{array}{r}2017 \text { Jun } 08 \\
02: 01: 16\end{array}$ & 520 & $340_{-140}^{+140}$ & $\mathrm{BH}-\mathrm{BH}$ & (d) & $\mathrm{HE}$ & & \multicolumn{2}{|c|}{$\begin{array}{l}{\left[T_{0}-60 \mathrm{~s}, T_{0}+60 \mathrm{~s}\right]} \\
\text { Out of FOV }\end{array}$} \\
\hline GW170814 & $\begin{array}{r}2017 \text { Aug } 14 \\
10: 30: 43\end{array}$ & 60 & $540_{-210}^{+130}$ & $\mathrm{BH}-\mathrm{BH}$ & (e) & $\mathrm{HE}$ & & \multicolumn{2}{|c|}{$\begin{array}{l}{\left[T_{0}-60 \mathrm{~s}, T_{0}+60 \mathrm{~s}\right]} \\
\text { Out of FOV }\end{array}$} \\
\hline GW170817 & $\begin{array}{r}2017 \text { Aug } 17 \\
12: 41: 04\end{array}$ & 28 & $40_{-14}^{+8}$ & NS-NS & (f) & $\mathrm{HE}$ & & \multicolumn{2}{|c|}{$\begin{array}{l}{\left[T_{0}-60 \mathrm{~s}, T_{0}+60 \mathrm{~s}\right]} \\
\text { Out of FOV }\end{array}$} \\
\hline
\end{tabular}

References. (a) Abbott et al. (2016c); (b) Abbott et al. (2016b); (c) Abbott et al. (2017a); (d) Abbott et al. (2017f); (e) Abbott et al. (2017b); (f) Abbott et al. (2017c).

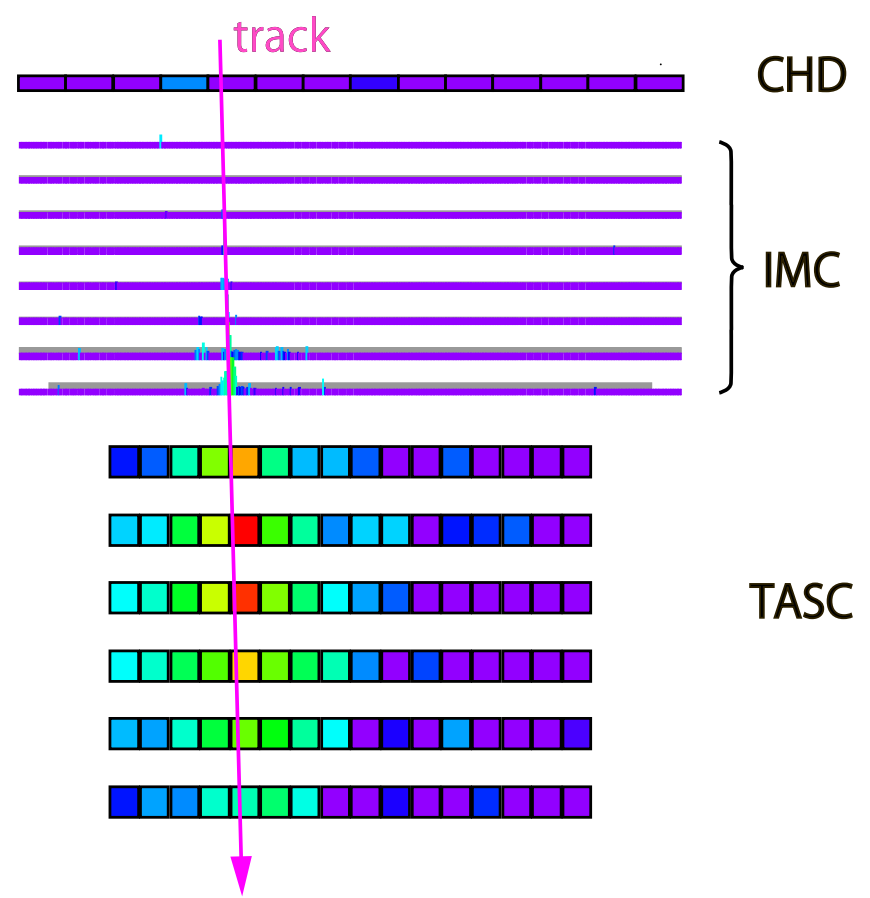

Figure 1. Schematic cross-sectional view of CALET/CAL with a sample event. We require that a track should cross the CHD (full area) and have at least a minimum path length in the TASC (see Cannady \& for the CALET Collaboration 2017, 2018 for details).

UT and turned off around 03:43 UT to avoid a high background radiation area. No CGBM on-board trigger was generated at the trigger time of GW151226. For other GW events (GW170104, GW170608, GW170814, and GW170817), CAL was collecting data in the HE mode since the ISS was in the high latitude region in its orbit. First results on the analysis of GW151226 have already been published (Adriani et al. 2016), and here we describe results with a refined analysis. We also give results on the comprehensive analysis of the CAL data for these four later events.

\subsection{Analysis of Gamma-Ray Events in CALET/CAL}

The selection process of gamma-ray events used for the HE mode is essentially the same as that described in Mori \& for the CALET Collaboration (2013). For the LE- $\gamma$ mode the selection and analysis are fully described in Cannady \& for the CALET Collaboration $(2017,2018)$. Here we briefly summarize the procedures.

Offline trigger - In order to remove the effects of variation in the hardware trigger threshold and gains in the flight data sample, energy deposit thresholds higher than those nominally applied by the hardware trigger are imposed offline both for LE- $\gamma$ and HE modes.

Tracking-Event tracks are reconstructed for the HE mode using the EM track algorithm (Akaike \& for the CALET Collaboration 2013) developed for the electron analysis, which is a powerful method for reconstructing electromagnetic showers. For the LE- $\gamma$ mode we use the CC track algorithm (Cannady \& for the CALET Collaboration 2017, 2018) optimized for photons with energies below $10 \mathrm{GeV}$. It begins by finding clusters of hit fibers in the three bottom layers of IMC separately for the $X$ - and $Y$-projections and extending the candidate tracks to the upper layers of IMC. The trajectory with the highest total energy deposit is selected. In the HE mode, contained events passing through the CHD with track lengths in TASC in excess of $26.4 \mathrm{~cm}$ are subjected to further analysis; in the LE- $\gamma$ mode, in order to maximize the FOV, we select well contained events whose tracks satisfy more sophisticated geometrical conditions (Cannady \& for the CALET Collaboration 2017, 2018).

Shower shape/hadronic rejection-Low-energy gamma-ray events can be mimicked by albedo (i.e., upward moving) secondary charged pions from hadronic interactions in the calorimeter or the support structure. These events are vetoed by requiring that more energy be deposited in the bottom IMC layer than in the layer where pair conversion occurs. Further rejection of events with showers not consistent with a pure electromagnetic cascade is provided by a cut on the IMC concentration, which uses the lateral spread of the energy deposit distribution in the lower layers of IMC. 


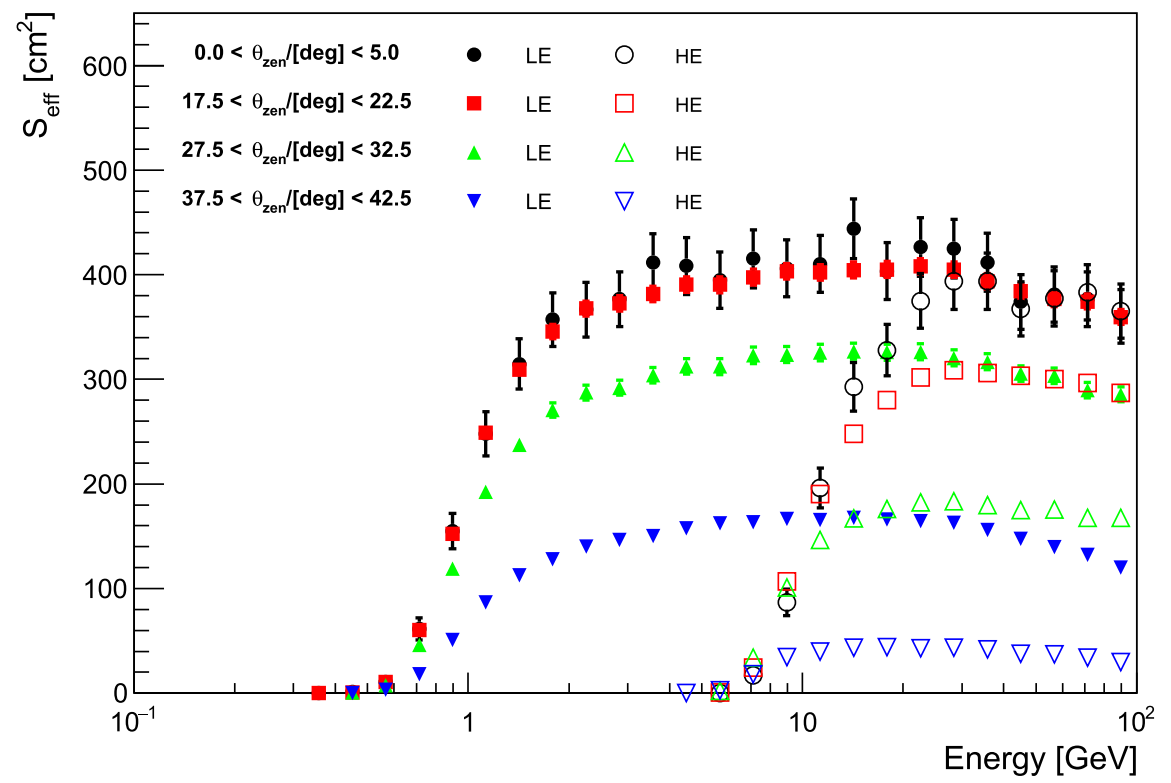

Figure 2. Effective area of $C A L E T / C A L$ as a function of gamma-ray energy in the low-energy gamma-ray mode (LE- $\gamma$ ) and high-energy mode (HE). Four ranges of incident zenith angles $\left(\theta_{\text {zen }}\right)$ are assumed. Statistical uncertainties due to Monte Carlo statistics are shown by error bars.

In order to reject hadronic events, we utilize the $K$ parameter defined as

$$
K=\log _{10} F_{E}+R_{E} / 2 \mathrm{~cm},
$$

where $F_{E}$ is the fractional energy deposit in the bottom TASC layer with respect to the total energy deposit sum in the TASC and $R_{E}$ is the second moment of the lateral energy deposit distribution in the top layer of TASC. This method is developed for the derivation of the electron flux and is designed to exploit the larger spread and slower development of proton showers due to penetrating secondary pions (Adriani et al. 2017).

Zero charge identification-In order to select events consistent with zero primary charge, cuts are made on the energy deposits in CHD and upper IMC layers. These requirements are designed to veto charged particle events effectively. We require one of three filters utilizing CHD and upper IMC layers (see Cannady \& for the CALET Collaboration 2017, 2018 for detail).

In addition, as described in detail in Cannady \& for the CALET Collaboration $(2017,2018)$, we have to reject gammaray candidate events that are generated in the ISS structures such as the Japanese Experiment Module to remove events generated in interactions of cosmic rays with these structures, which create gamma-ray event clusters clearly seen in our FOV. After these selections, incident gamma-ray energies are derived from the deposited energies based on Monte Carlo simulations, pre-flight accelerator calibrations, and in-flight noninteracting penetrating particle events.

Based on the CALET simulation studies (Mori \& for the CALET Collaboration 2013; Cannady \& for the CALET Collaboration 2017, 2018), the gamma-ray efficiency reaches its maximum around $10 \mathrm{GeV}$ with an efficiency of $48 \%$ relative to an area of the TASC top layer (excluding a $1.9 \mathrm{~cm}$ margin around the outside) for normal incidence, after applying the event selections described above. This figure is to be compared with a pair creation probability of $54 \%$ in 1 radiation length, which is approximately the thickness required to be tracked in at least three layers in IMC, and implies a high efficiency in the gamma-ray event reconstruction and selection processes. The effective areas for four ranges of incident angles are shown in Figure 2 as a function of gamma-ray energy.

The GeV sky is rather bright along the Galactic plane due to the Galactic diffuse gamma-ray radiation with Galactic and extragalactic individual sources, and there is a residual all-sky emission component called the isotropic diffuse gamma-ray background. These gamma-rays are a source of background in a search for gamma-ray emission associated with $\mathrm{GW}$ events. The expected number of background events in the time window used in our search was calculated using a prediction based on the Fermi-LAT Pass 8 measurements. ${ }^{48}$ As shown by Cannady \& for the CALET Collaboration $(2017,2018)$, the CALET measurement is in reasonable agreement with the LAT result.

The upper limit of the CAL observation in the time windows is estimated as follows: first, we calculate the effective area as a function of gamma-ray energy, and the resultant energydependent exposure map in the time window for the corresponding energy region depending on the trigger mode (LE- $\gamma$ or HE). In the case of a null event, we estimate the upper limit on the gamma-ray flux corresponding to 2.44 events (the $90 \%$ confidence limit for a null observation) assuming a powerlaw spectrum with a single photon index of -2 by using the calculated exposure map. The photon index, -2 , is taken as a typical value for Fermi-LAT GRBs in the GeV energy range (Ackermann et al. 2013).

\subsection{GW151226 $6^{49}$}

We searched for gamma-ray events associated with GW151226 using the CAL data in the time window $\left[T_{0}-525 \mathrm{~s}, T_{0}+211 \mathrm{~s}\right]$ around the LIGO trigger time $\left(T_{0}\right)$,

\footnotetext{
${ }^{48}$ We utilized a gamma-ray sky map in the energy range of $1-100 \mathrm{GeV}$ created using the archival data for the dates 2008-08-04 through 2017-03-12 available via https://fermi.gsfc.nasa.gov/ssc/data/access/lat/.

49 The result shown here is an improved version based on more refined analysis compared with that presented in our previous paper (Adriani et al. 2016).
} 


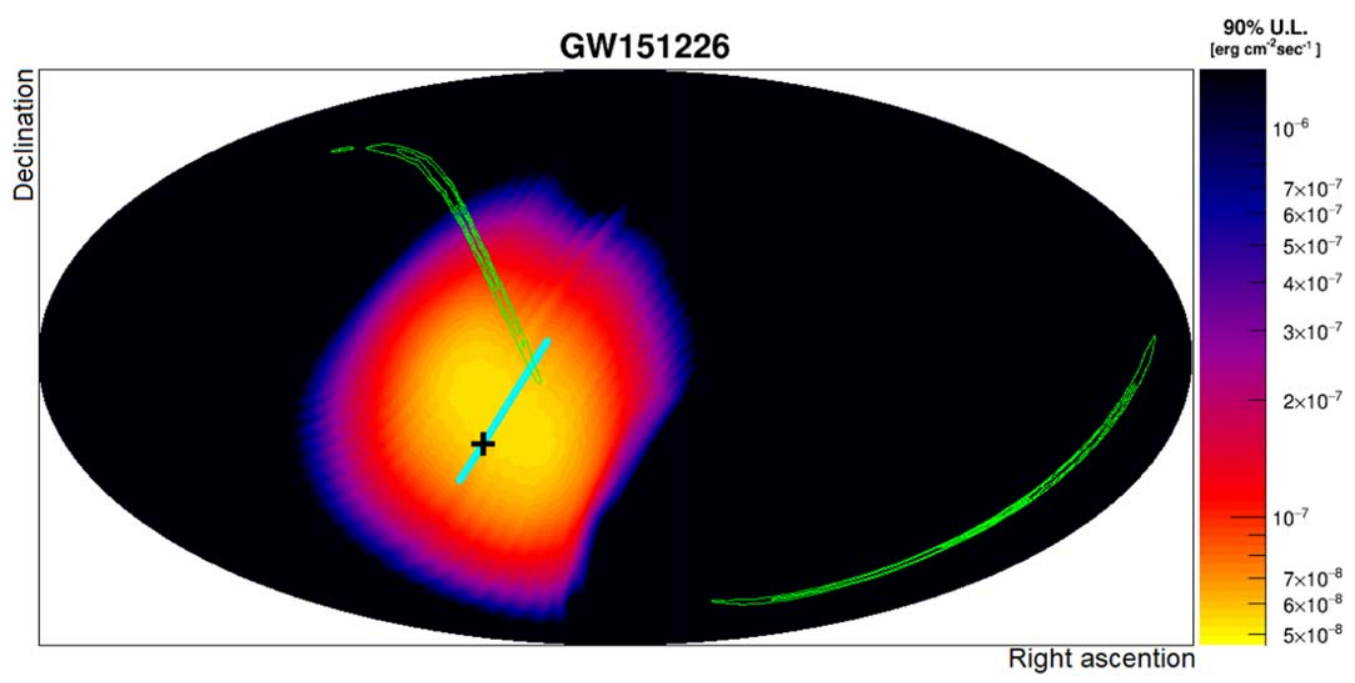

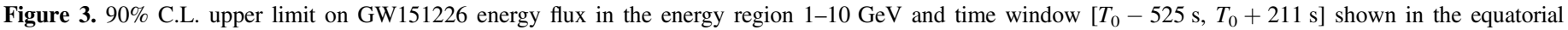

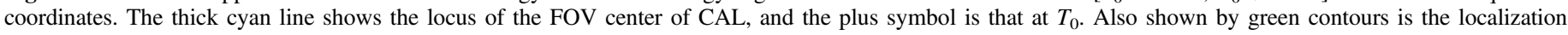
significance map of the GW151226 signal reported by LIGO.

the time period when the CAL was operational in the LE- $\gamma$ mode with an energy threshold of $1 \mathrm{GeV}$. We analyzed the full length of this window in order to perform the most sensitive search possible with increased statistics.

Expected number of contaminated background gamma-rays is small because the searched area of the sky for the GW151226 counterpart is significantly apart from the Galactic plane. In fact, the number of expected background events is 0.051 in this time window for the sky region covering $25 \%$ of the summed LIGO probabilities; i.e., the CAL observation is almost background-free for such a short time period. No candidates were found in this time window and sky region, resulting in an upper limit that is calculated as described in the previous section.

Figure 3 shows the sky map of the 90\%-confidence-level upper limit on the gamma-ray flux. The estimated upper limit is $9.3 \times 10^{-8} \mathrm{erg} \mathrm{cm}^{-2} \mathrm{~s}^{-1}(90 \%$ C.L.) in the $1-10 \mathrm{GeV}$ region where the coverage of CAL reaches $15 \%$ of the integrated LIGO probability $(\sim 1.1 \mathrm{sr})$. If we enlarge the sky region to contain $25 \%$ of the LIGO integrated probability, the upper limit is $2.8 \times 10^{-7} \mathrm{erg} \mathrm{cm}^{-2} \mathrm{~s}^{-1}$ in the same energy region. The luminosity upper limit set by CAL is estimated as $2.3(6.8) \times$ $10^{48} \mathrm{erg} \mathrm{s}^{-1}$ assuming a luminosity distance of $440 \mathrm{Mpc}$ for coverage of $\sim 15(25) \%$ of the LIGO integrated probability regions. By comparison, the upper limit in the energy flux in the $0.1-1 \mathrm{GeV}$ region as reported by Fermi-LAT (assuming a power-law spectrum with a single photon index of -2) is $3 \times 10^{-10} \mathrm{erg} \mathrm{cm}^{-2} \mathrm{~s}^{-1}$ (95\% C.L.) for the time window $\left[T_{0}, T_{0}+1 \times 10^{4} \mathrm{~s}\right]$ (Racusin et al. 2017), corresponding to $\sim 4 \times 10^{-9} \mathrm{erg} \mathrm{cm}^{-2} \mathrm{~s}^{-1}$ for the $736 \mathrm{~s}$ time window of the CAL in the LE $\gamma$ mode for this GW event.

We also calculate upper limits on energy flux of gamma-rays in smaller time windows since we do not know the time profile of the possible electromagnetic emission that accompanies gravitational wave events. When we set the window as $\left[T_{0}-60 \mathrm{~s}, T_{0}+60 \mathrm{~s}\right]$, the upper limit in the $1-10 \mathrm{GeV}$ region is $9.4(20) \times 10^{-7} \mathrm{erg} \mathrm{cm}^{-2} \mathrm{~s}^{-1}$ for the integrated LIGO probabilities inside the CAL FOV of $\sim 15(25) \%$. If we set the window as $\left[T_{0}-1 \mathrm{~s}, T_{0}+1 \mathrm{~s}\right]$, the upper limit in the $1-10 \mathrm{GeV}$ region is $5.3 \times 10^{-5} \mathrm{erg} \mathrm{cm}^{-2} \mathrm{~s}^{-1}$ for the LIGO integrated probabilities in the CAL FOV at the level of $\sim 15 \%$.

\subsection{GW170104}

For the time period around the trigger time $\left(T_{0}\right)$ corresponding to GW170104, CAL was running in the HE mode with an energy threshold of $10 \mathrm{GeV}$. Gamma-ray events have been searched for using the CAL data in the time window $\left[T_{0}-60 \mathrm{~s}, T_{0}+60 \mathrm{~s}\right]$ but no candidates were found. The estimated number of background events expected in this time window is $7.8 \times 10^{-4}$. We calculated an upper limit on the gamma-ray energy flux of $6.4 \times 10^{-6} \mathrm{erg} \mathrm{cm}^{-2} \mathrm{~s}^{-1}$ at $90 \%$ C.L. in the $10-100 \mathrm{GeV}$ energy region for the sky region covering $30 \%$ of the integrated LIGO probabilities (Figure 4). This upper limit corresponds to $6.2 \times 10^{50} \mathrm{erg} \mathrm{s}^{-1}$ assuming a luminosity distance of $880 \mathrm{Mpc}$. If we set a narrower time window as $\left[T_{0}-1, T_{0}+1 \mathrm{~s}\right]$, the estimated number of background events is $1.2 \times 10^{-5}$ and the upper limit is $4.3 \times 10^{-4} \mathrm{erg} \mathrm{cm}^{-2} \mathrm{~s}^{-1}$ for the flux and $4.1 \times 10^{52} \mathrm{erg} \mathrm{s}^{-1}$ for the luminosity (90\% C.L.) assuming the same sky region.

We note that $A G I L E$ reported a weak $(4.4 \sigma)$ event lasting about $32 \mathrm{~ms}$ and occurring $0.46 \pm 0.05 \mathrm{~s}$ before $T_{0}$ in the omni-directional MCAL data in the $0.4-100 \mathrm{MeV}$ region (Verrecchia 2017a), while other searches for high-energy emission yielded upper limits only.

\subsection{GW170608}

For the time period around the trigger time $\left(T_{0}\right)$ corresponding to GW170608, CAL was running in the HE mode with an energy threshold of $10 \mathrm{GeV}$. Gamma-ray events have been searched for using the CAL data in the time window $\left[T_{0}-60 \mathrm{~s}, T_{0}+60 \mathrm{~s}\right]$ but no candidates were found. Unfortunately, the sky coverage of CAL did not include the region of the localization $\left(520 \mathrm{deg}^{2}\right)$ determined with two interferometric detectors as shown in Figure 5.

We note that Fermi-LAT reported a weak $(3.5 \sigma)$ excess around the LIGO location area in the $\left[T_{0}, T_{0}+1 \mathrm{ks}\right]$ window in the energy region above $100 \mathrm{MeV}$ (Omodei 2017), but 


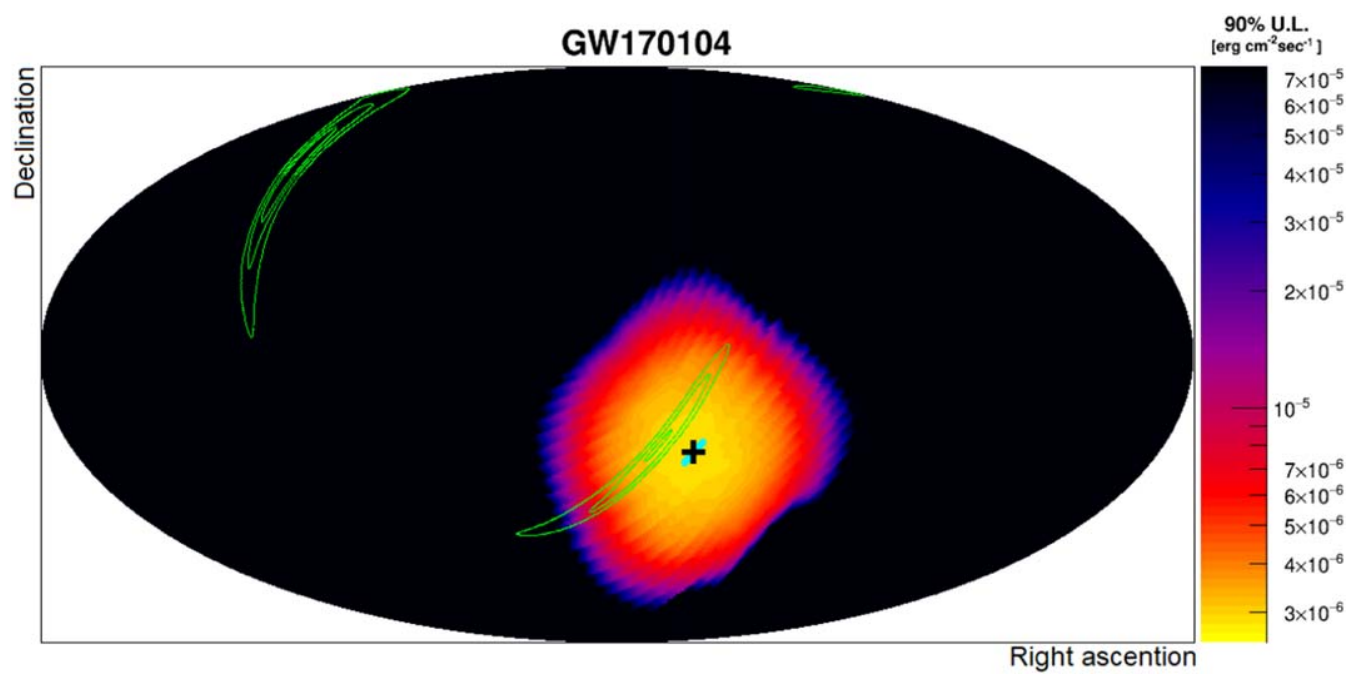

Figure 4. $90 \%$ C.L. upper limit on GW170104 energy flux in the energy region 10-100 GeV and time window $\left[T_{0}-60 \mathrm{~s}, T_{0}+60 \mathrm{~s}\right]$ shown in the equatorial coordinates. Thick cyan line shows the locus of the FOV center of CAL, and the plus symbol is that at $T_{0}$. Also shown by green contours is the localization significance map of the GW170104 signal reported by LIGO.

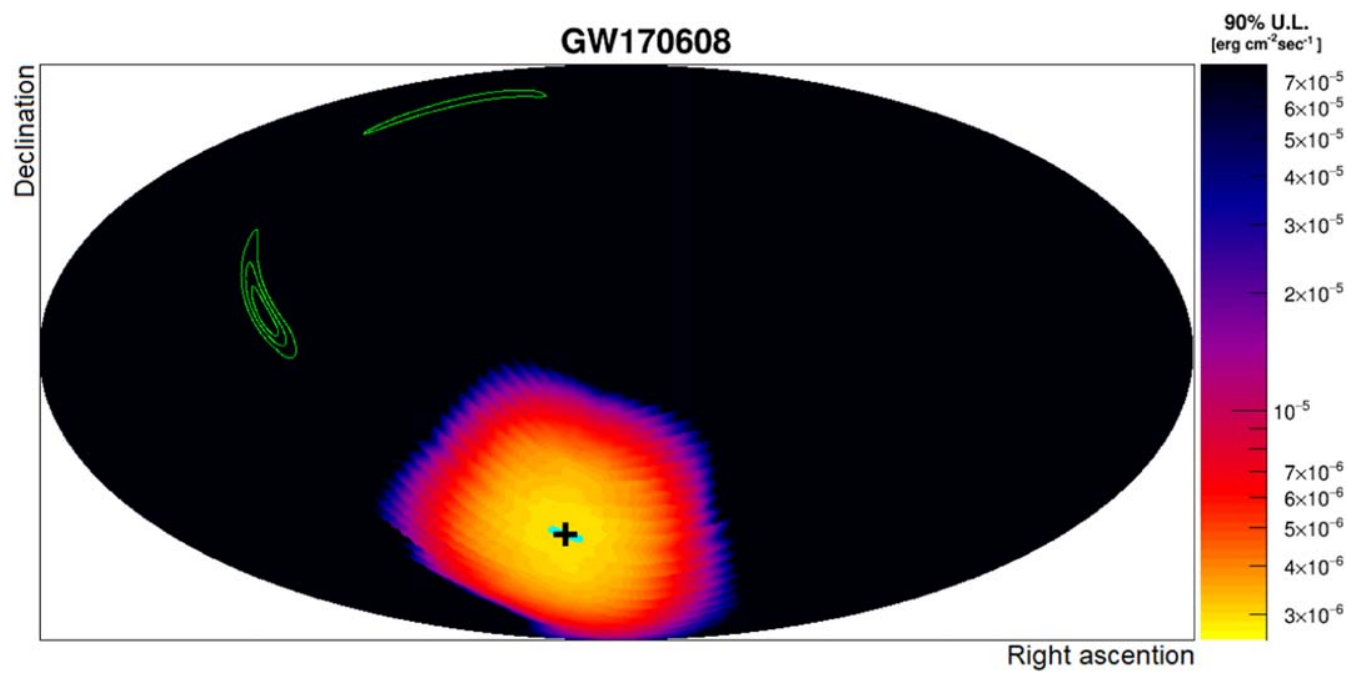

Figure 5. $90 \%$ C.L. upper limit on GW170608 energy flux in the energy region $10-100 \mathrm{GeV}$ and time window $\left[T_{0}-60 \mathrm{~s}, T_{0}+60 \mathrm{~s}\right]$ shown in the equatorial coordinates. Thick cyan line shows the locus of the FOV center of CAL, and the plus symbol is that at $T_{0}$. Also shown by green contours is the localization significance map of the GW170608 signal reported by LIGO.

others reported only upper limits for high-energy emission for this GW event.

\subsection{GW170814}

For the time period around the trigger time $\left(T_{0}\right)$ corresponding to GW170814, CAL was running in the HE mode with an energy threshold of $10 \mathrm{GeV}$. Gamma-ray events have been searched for using CAL data in the time window $\left[T_{0}-60 \mathrm{~s}, T_{0}+60 \mathrm{~s}\right]$ but no candidates were found. Unfortunately, the sky coverage of CAL did not include the rather small region of the localization $\left(60 \mathrm{deg}^{2}\right)$ determined with three interferometric detectors, as shown in Figure 6.

We note that INTEGRAL/SPI-ACS reported a weak $3.5 \sigma$ excess in the $\left[T_{0}-1.5 \mathrm{~s}, T_{0}+8.5 \mathrm{~s}\right]$ window (Pozanenko 2017), but this was not confirmed by an independent analysis (Savchenko 2017). Other reports gave only upper limits on high-energy emission for this GW event.

\section{7. $G W 170817$}

$1.7 \mathrm{~s}$ after the trigger due to the LIGO-Virgo event GW170817 $\left(T_{0}\right)$, Fermi-GBM and INTEGRAL detected GRB $170817 \mathrm{~A}$ with $T_{90}$ duration $2.0 \pm 0.5 \mathrm{~s}$ (Abbott et al. 2017d). For the time period around GW170817, CAL was running in the $\mathrm{HE}$ mode with an energy threshold of $10 \mathrm{GeV}$. Gamma-ray events have been searched for using the CAL data in the time window $\left[T_{0}-60 \mathrm{~s}, T_{0}+60 \mathrm{~s}\right]$ but no candidates were found. Unfortunately, the sky coverage of CAL did not include the rather small region of the localization $\left(28 \mathrm{deg}^{2}\right)$ determined with three interferometric detectors, as shown in Figure 7. It has been reported that the gravitational wave signal started about $100 \mathrm{~s}$ before $T_{0}$, but it was also out of the FOV of CAL during this period.

We have also searched for possible delayed signal from this merger event (Murase et al. 2018). In the two-month period (2017 August 17-October 16) after the event we had no gamma-ray candidate around the direction of its counterpart 


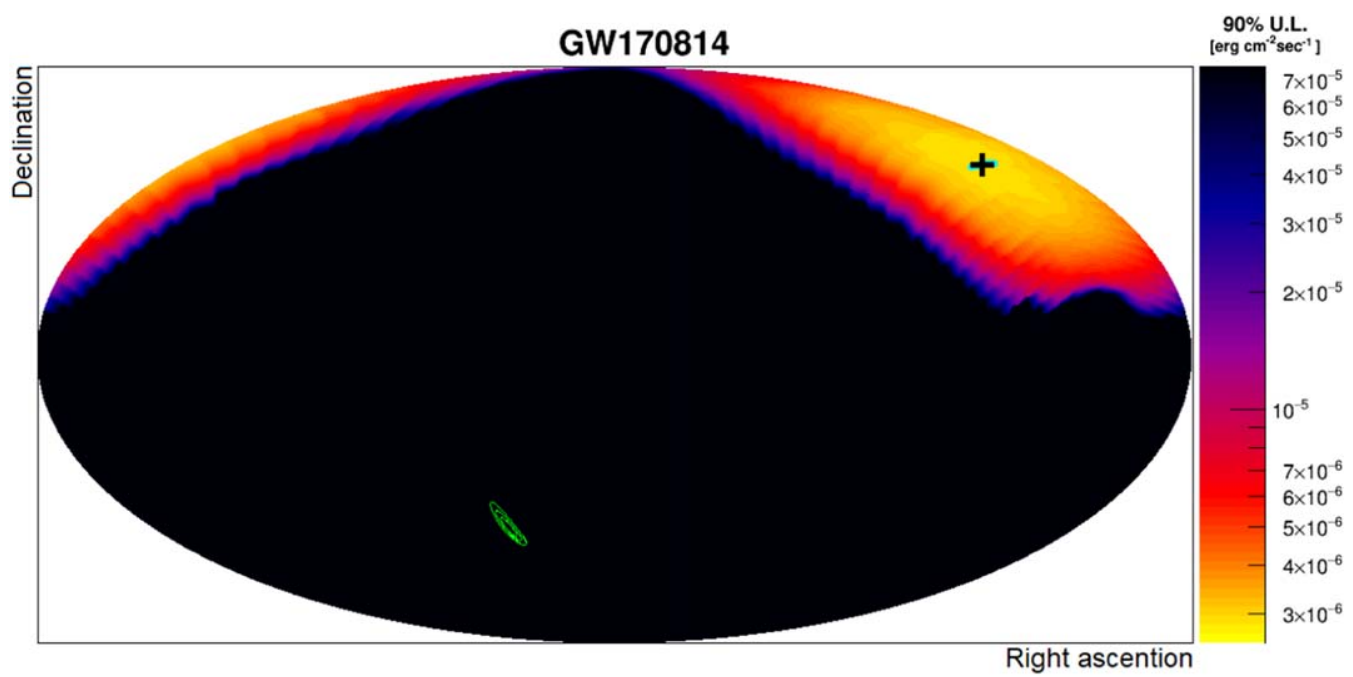

Figure 6. $90 \%$ C.L. upper limit on GW170814 energy flux in the energy region 10-100 GeV and time window $\left[T_{0}-60 \mathrm{~s}, T_{0}+60 \mathrm{~s}\right]$ shown in the equatorial coordinates. Thick cyan line shows the locus of the FOV center of CAL, and the plus symbol is that at $T_{0}$. Also shown by green contours is the localization significance map of the GW170814 signal reported by LIGO/Virgo.

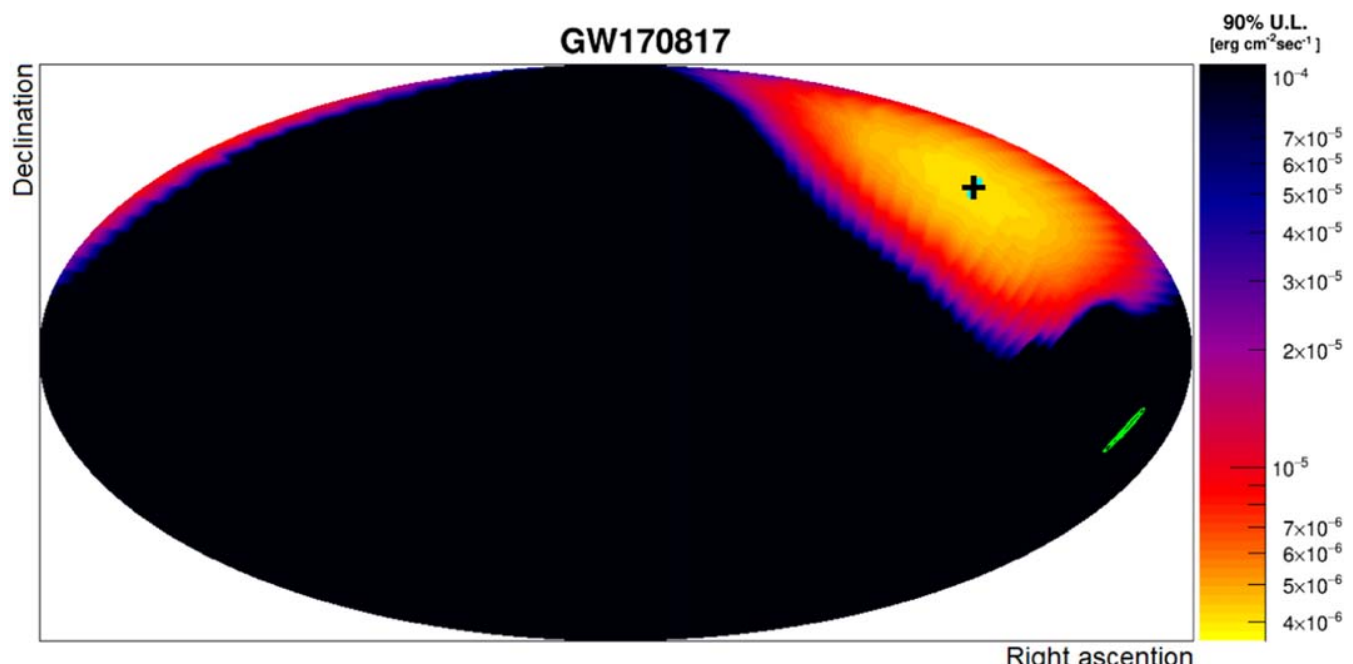

Figure 7. $90 \%$ C.L. upper limit on GW170817 energy flux in the energy region $10-100 \mathrm{GeV}$ and time window $\left[T_{0}-60 \mathrm{~s}, T_{0}+60 \mathrm{~s}\right]$ shown in the equatorial coordinates. Thick cyan line shows the locus of the FOV center of CAL, and the plus symbol is that at $T_{0}$. Also shown by green contours is the localization significance map of the GW170817 signal reported by LIGO/Virgo.

object (NGC 4993) (Abbott et al. 2017e), and obtained 90\% C. L. upper limits on the energy flux of $1.2 \times 10^{-10} \mathrm{erg} \mathrm{cm}^{-2} \mathrm{~s}^{-1}$ $\left(4.0 \times 10^{-10} \mathrm{erg} \mathrm{cm}^{-2} \mathrm{~s}^{-1}\right)$ for gamma-rays above $1 \mathrm{GeV}$ $(10 \mathrm{GeV})$ using the LE- $\gamma$ mode (the HE mode). This upper limit corresponds to $2.4 \times 10^{43} \mathrm{erg} \mathrm{s}^{-1}\left(8.0 \times 10^{43} \mathrm{erg} \mathrm{s}^{-1}\right)$ assuming a luminosity distance of $40 \mathrm{Mpc}$.

\section{Future Prospects}

Identifying the electromagnetic counterpart of a gravitational wave event would be a key discovery to constrain the origin of the event. The detection of multiwavelength radiation in association with GW170817 (Abbott et al. 2017e) was a huge step to open a new window of astronomy. In particular the detection of a gamma-ray burst, GRB 170817A, observed $\sim 1.7 \mathrm{~s}$ after GW170817 by Fermi-GBM and INTEGRAL (Abbott et al. 2017d), provides new insight into the origin of short gamma-ray bursts. The association of GW170817 and GRB 170817A can be interpreted as a merger of a neutron starneutron star binary, which is hypothesized to be a possible origin of short gamma-ray bursts as discussed in Section 1. However, GRB 170817A, which is the closest short GRB ever observed, is 2-6 orders of magnitude less energetic than other bursts with known distances.

The underluminous nature of GRB 170817A may imply that the gamma-rays detected with Fermi-GBM are off-axis emission from a typical short GRB (Abbott et al. 2017c; Alexander et al. 2017; Margutti et al. 2017; Troja et al. 2017; Ioka \& Nakamura 2018; Lazzati et al. 2018). Although the $(50-300 \mathrm{keV}) /(10-50 \mathrm{keV})$ hardness ratio is small ("soft"), the spectral peak energy is close to the lower end of the typical value in spite of the off-axis observation (but see Kisaka et al. 2017 for an example). The rising X-ray and radio afterglow light curves as far as 100 days (Mooley et al. 2017; Ruan et al. 2017) are also difficult to explain with an off-axis afterglow model with a simple top-hat jet (or they may suggest a structured jet). GRB 170817A may belong to another population of gamma-ray transient phenomena other than the short GRB as proposed by Bromberg et al. (2018), Gottlieb 


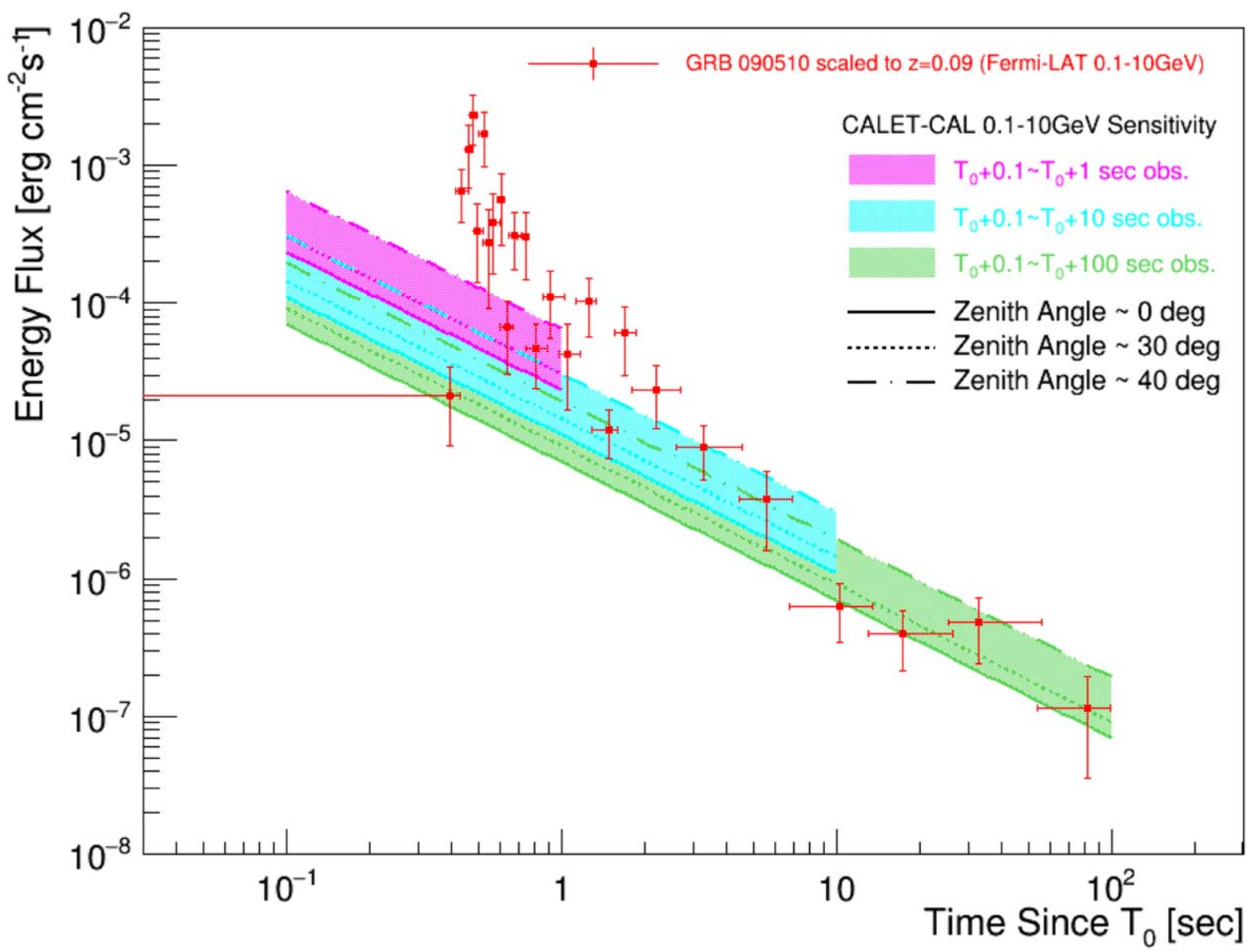

Figure 8. CALET/CAL sensitivity to obtain 1 event for a transient source assuming the energy spectrum proportional to $E^{-2} t^{-1}$, where $E$ is the energy and $t$ is the time after $T_{0}$, in the energy region $0.1-10 \mathrm{GeV}$. Despite the lack of sensitivity to sub-GeV gamma-rays in the CAL, the $0.1-1 \mathrm{GeV}$ band is included in this calculation of the limit to compare to the Fermi-LAT light curve since the energy flux is sensitive to the range over which it is integrated. Shaded areas show energy-flux sensitivities assuming observations of 1,10 , and $100 \mathrm{~s}$ durations for a source around the zenith, and dotted and dotted-dashed lines show those for a source around $30^{\circ}$ and $40^{\circ}$ from zenith, respectively. Also shown by points are the observed light curve of GRB 090510 by Fermi-LAT, which is a short-hard GRB with an additional hard power-law component from $10 \mathrm{keV}$ to GeV energies (Ajello et al. 2018), scaled to $z=0.09$, the nominal redshift of the first LIGO event GW150914 as calculated by Ackermann et al. (2016).

et al. (2018), Kasliwal et al. (2017), Murguia-Berthier et al. (2017), and Asano \& To (2018). In such cases, the expected gamma-ray flux in the $\mathrm{GeV}$ range is not constrained by the previous short GRB observations.

The fluence of GRB 170817A in the keV-MeV energy band was observed to be $(1.4 \pm 0.3) \times 10^{-7} \mathrm{erg} \mathrm{cm}^{-2}$ (Abbott et al. 2017c). Had the same level of fluence been present in the $\mathrm{GeV}$ energy band, it could have be detected by $\mathrm{GeV}$ gamma-ray detectors in operation at that time. However, Fermi-LAT was entering the South Atlantic Anomaly and was not collecting data until about $10^{3} \mathrm{~s}$ after GRB 170817A (Ajello et al. 2018), and AGILE started observation after about $10^{3} \mathrm{~s}$ (Verrecchia et al. 2017b). It was also out of the FOV of CALET as reported above. Thus, unfortunately, there is no limit in the $\mathrm{GeV}$ band around the trigger time of GW170817.

Regarding sGRB events in general, some events have been observed to emit high-energy ( $>100 \mathrm{MeV}$ ) gamma-rays (e.g., GRB 081024B, Abdo et al. 2010; and GRB 090510, Ackermann et al. 2010). Their fluence in the high-energy band could be comparable to that in the hard X-ray band (Abdo et al. 2010). However, the fraction of GRBs showing high-energy emission observed to date is fairly low (Ackermann et al. 2013). This could be due to intrinsic properties associated with the gamma-ray emission mechanism, but another reason could be the limited FOV of GeV gamma-ray detectors $(2 \sim 3 \mathrm{sr})$.

Figure 8 shows the sensitivity of $C A L E T / \mathrm{CAL}$ to obtain 1 event assuming an observation of 1,10 , and $100 \mathrm{~s}$ durations. The typical energy range of the on-axis gamma-ray emission from NS-NS mergers can be higher than that of the short GRB emission from GRB 170817A. Although the effective area of CALET/CAL is smaller than that of Fermi-LAT, the fields of view of the two detectors are comparable. As the sensitivity of laser interferometers is expected to increase in coming years, the number of gamma-ray transients associated with GW events falling into the possible new population mentioned above will also increase. If their spectra extend to $\mathrm{GeV}$ energies, they could be easily detectable as shown in Figure 8. Thus $C A L E T / \mathrm{CAL}$ could contribute to constrain the $\mathrm{GeV}$ emission from a nearby NS-NS merger simultaneously with a GW signal in the near future. Monitoring the $\mathrm{GeV}$ sky with CALET, with its mission scheduled to continue for three more years, may complement the coverage by other missions and may help to study unexplored highenergy emission from future transient events.

We would like to thank the anonymous referee for comments and suggestions that materially improved the paper. We 
gratefully acknowledge JAXA's contributions for CALET development and operation on ISS. We express our sincere thanks to ASI and NASA for their support to the CALET project. This work is partially supported by JSPS Grant-in-Aid for Scientific Research (S) Number 26220708, JSPS Grant-inAid for Scientific Research (B) Number 17H02901, JSPS Grant-in-Aid for Scientific Research (C) Number 16K05382, and MEXT-Supported Program for the Strategic Research Foundation at Private Universities (2011-2015) S1101021 in Waseda University. This work is also supported in part by MEXT Grant-in-Aid for Scientific Research on Innovative Areas Number 24103002. US CALET work is supported by NASA under RTOP 14-APRA14-0075 (GSFC) and grants NNX16AC02G (WUStL), NNX16AB99G (LSU), and NNX11AE06G (Denver).

\section{ORCID iDs}

O. Adriani (ㄴ) https://orcid.org/0000-0002-3592-0654

K. Asano (1) https://orcid.org/0000-0001-9064-160X

Y. Asaoka (1) https://orcid.org/0000-0001-6440-933X

W. R. Binns (i) https://orcid.org/0000-0001-6110-3407

M. Bongi (i) https://orcid.org/0000-0002-6050-1937

M. L. Cherry (1) https://orcid.org/0000-0003-2808-312X

V. Di Felice (1) https://orcid.org/0000-0002-6404-6177

K. Ebisawa (10 https://orcid.org/0000-0002-5352-7178

K. Ioka (1) https://orcid.org/0000-0002-3517-1956

M. H. Israel (i) https://orcid.org/0000-0002-8104-208X

K. Kasahara (1) https://orcid.org/0000-0001-5611-3301

J. Kataoka ๑ https://orcid.org/0000-0003-2819-6415

R. Kataoka (1) https://orcid.org/0000-0001-9400-1765

N. Kawanaka (1) https://orcid.org/0000-0001-8181-7511

H. S. Krawczynski (1) https://orcid.org/0000-0002-1084-6507

M. Mori $\odot$ https://orcid.org/0000-0003-2921-1592

N. Mori (1) https://orcid.org/0000-0003-2138-3787

K. Munakata 는 https://orcid.org/0000-0002-2131-4100

S. B. Ricciarini (1) https://orcid.org/0000-0001-6176-3368

R. Sparvoli (1) https://orcid.org/0000-0002-6314-6117

J. E. Suh (1) https://orcid.org/0000-0003-1024-2292

Y. Tsunesada (1) https://orcid.org/0000-0001-9238-6817

\section{References}

Abbott, B. P., Abbott, R., Abbott, T. D., et al. 2016a, PhRvL, 116, 061102 Abbott, B. P., Abbott, R., Abbott, T. D., et al. 2016b, PhRvL, 116, 241103 Abbott, B. P., Abbott, R., Abbott, T. D., et al. 2016c, PhRvX, 6, 041015 Abbott, B. P., Abbott, R., Abbott, T. D., et al. 2017a, PhRvL, 118, 221101 Abbott, B. P., Abbott, R., Abbott, T. D., et al. 2017b, PhRvL, 119, 141101 Abbott, B. P., Abbott, R., Abbott, T. D., et al. 2017c, PhRvL, 119, 161101 Abbott, B. P., Abbott, R., Abbott, T. D., et al. 2017d, ApJL, 848, L13
Abbott, B. P., Abbott, R., Abbott, T. D., et al. 2017e, ApJL, 848, L12 Abbott, B. P., Abbott, R., Abbott, T. D., et al. 2017f, ApJL, 851, L35 Abdo, A. A., Ackermann, M., Ajello, M., et al. 2010, ApJ, 712, 558 Acero, F., Ackermann, M., Ajello, M., et al. 2016, ApJS, 223, 26 Ackermann, M., Ajello, M., Albert, A., et al. 2016, ApJL, 823, L2 Ackermann, M., Ajello, M., Asano, K., et al. 2013, ApJS, 209, 11 Ackermann, M., Asano, K., Atwood, W. B., et al. 2010, ApJ, 716, 1178 Adriani, O., Akaike, Y., Asano, K., et al. 2016, ApJL, 829, L20

Adriani, O., Akaike, Y., Asano, K., et al. 2017, PhRvL, 119, 181101 Ajello, M., Allafort, A., Axelsson, M., et al. 2018, ApJ, 861, 85

Akaike, Y. \& for the CALET Collaboration 2013, Proc. ICRC (Rio de Janeiro), 0726,2162

Alexander, K. D., Berger, E., Fong, W., et al. 2017, ApJL, 848, L21

Asano, K., \& To, S. 2018, ApJ, 852, 105

Asaoka, Y., Akaike, Y., Komiya, Y., et al. 2017, APh, 91, 1

Asaoka, Y., Ozawa, S., Torii, S., et al. 2018, APh, 100, 29

Bromberg, O., Tchekhovskoy, A., Gottlieb, O., Nakar, E., \& Piran, T. 2018, MNRAS, 475, 2971

Cannady, N. \& for the CALET Collaboration 2017, Proc. ICRC (Busan), 35,720

Cannady, N. \& for the CALET Collaboration 2018, ApJS, in press

de Mink, S. E., \& King, A. 2017, ApJL, 839, L7

Eichler, D., Livio, M., Piran, T., \& Schramm, D. N. 1989, Natur, 340, 126

Einstein, A. 1916, SPAW, 688

Einstein, A. 1918, SPAW, 154

Fernández, R., \& Metzger, B. D. 2016, ARNPS, 66, 23

Goodman, J. 1986, ApJL, 308, L47

Gottlieb, O., Nakar, E., Piran, T., \& Hotokezaka, K. 2018, MNRAS, 479,588

Ioka, K., \& Nakamura, T. 2018, PTEP, 043E02

Kasliwal, M. M., Nakar, E., Singer, L. P., et al. 2017, Sci, 358, 1559

Kisaka, S., Ioka, K., Kashiyama, K., \& Nakamura, T. 2017, arXiv:1711.00243

Lazzati, D., Perna, R., Morsony, B. J., et al. 2018, PhRvL, 120, 241103

Margutti, R., Berger, E., Fong, W., et al. 2017, ApJL, 848, L20

Mochkovitch, R., Hernanz, J., Isern, J., \& Martin, X. 1993, Natur, 361, 236

Mooley, K. P., Nakar, E., Hotokezaka, K., et al. 2017, Natur, 554, 207

Mori, M. \& for the CALET Collaboration 2013, Proc. ICRC (Rio de Janeiro), 0248,1185

Murase, K., Toomey, M. W., Fang, K., et al. 2018, ApJ, 854, 60

Murguia-Berthier, A., Ramirez-Ruiz, E., Kilpatrick, C. D., et al. 2017, ApJL, 848, L34

Narayan, R., Paczynsky, B., \& Piran, T. 1992, ApJL, 395, L83

Omodei, N. 2017, GCN, 21227

Pacyński, B. 1986, ApJL, 308, L43

Phinney, E. S. 2009, New Worlds, New Horizons in Astronomy and Astrophysics (Washington, D.C.: The National Academies Press)

Pozanenko, A. 2017, GCN, 21476

Racusin, J. L., Burns, E., Goldstein, A., et al. 2017, ApJ, 835, 82

Rosswog, S. 2015, Int. J. Mod. Phys., 24, 1530012

Ruan, J. J., Nynka, M., Haggard, D., Kalogera, V., \& Evans, P. 2018, ApJL, 859, L16

Savchenko, V. 2017, GCN, 21478

Torii, S. \& for the CALET Collaboration 2015, Proc. ICRC (Hague), 34, 581

Troja, E., Piro, L., van Eerten, H., et al. 2017, Natur, 551, 71

Verrecchia, F., Tavani, M., Donnarumma, I., et al. 2017b, ApJL, 850, L27

Verrecchia, F., Tavani, M., Ursi, A., et al. 2017a, ApJL, 847, L20

Yamaoka, K. \& for the CALET Collaboration 2013, arXiv:1311.4084

Yamaoka, K. \& for the CALET Collaboration 2017, Proc. ICRC, 35, 614 